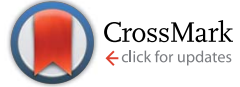

Cite this: Chem. Sci., 2015, 6, 1301

Received 2nd October 2014

Accepted 18th November 2014

DOI: $10.1039 / c 4 s c 03027 c$

www.rsc.org/chemicalscience

\title{
A novel copper-chelating strategy for fluorescent proteins to image dynamic copper fluctuations on live cell surfaces $\dagger$
}

\begin{abstract}
Yoon-Aa Choi, ${ }^{a}$ Joo Oak Keem, ${ }^{a}$ Cha Yeon Kim, ${ }^{b}$ Hye Ryeon Yoon, ${ }^{c}$ Won Do Heo, ${ }^{d}$ Bong Hyun Chung*a and Yongwon Jung ${ }^{\star c}$

Copper is indispensable in most aerobic organisms although it is toxic if unregulated as illustrated in many neurodegenerative diseases. To elucidate the mechanisms underlying copper release from cells, a membrane-targeting reporter which can compete with extracellular copper-binding molecules is highly desirable. However, engineering a reporter protein to provide both high sensitivity and selectivity for copper(॥) has been challenging, likely due to a lack of proper copper(॥)-chelating strategies within proteins. Here, we report a new genetically encoded fluorescent copper(II) reporter by employing a copper-binding tripeptide derived from human serum albumin (HSA), which is one of the major copperbinding proteins in extracellular environments. Optimized insertion of the tripeptide into the green fluorescent protein leads to rapid fluorescence quenching (up to $>85 \%$ change) upon copper-binding, while other metal ions have no effect. Furthermore, the high binding affinity of the reporter enables reliable copper detection even in the presence of competing biomolecules such as HSA and amyloid beta peptides. We also demonstrate that our reporter proteins can be used to visualize dynamic copper fluctuations on living HeLa cell surfaces.
\end{abstract}

\section{Introduction}

Copper is an essential metal ion in most aerobic organisms, participating in many critical biological processes including energy generation, oxygen transport, cellular metabolism and signal transduction. ${ }^{\mathbf{1}}$ However, when unregulated, copper can become toxic by generating reactive oxygen species (ROS) via Fenton-type reactions. ${ }^{2}$ The adverse effect of dysregulated copper homeostasis in humans is well illustrated in Menkes and Wilson's diseases, which are related to copper deficiency or overload, respectively. ${ }^{3}$ To address copper transport and trafficking in living systems, a variety of synthetic and genetically encoded probes have been developed with a focus mainly on visualizing intracellular copper, ${ }^{4}$ where $\mathrm{Cu}^{+}$is the major form due to reduction upon cellular uptake and the reducing environment of the cytosol. ${ }^{5}$

${ }^{a}$ BioNano Health Guard Research Center, 125 Gwahak-ro, Yuseong-gu, Daejeon, 305-806, Republic of Korea. E-mail: chungbh@kribb.re.kr; Fax: +82-42-860-4209; Tel: $+82-42-860-4442$

${ }^{b}$ Graduate School of Nanoscience and Technology, Korea Advanced Institute of Science and Technology (KAIST), Republic of Korea

${ }^{c}$ Department of Chemistry, KAIST, 291 Daehak-ro, Yuseong-gu, Daejeon, 305-701, Republic of Korea. E-mail: ywjung@kaist.ac.kr; Fax: +82-42-350-2810; Tel: +82-42350-2817

${ }^{d}$ Department of Biological Sciences, KAIST, Republic of Korea

$\dagger$ Electronic supplementary information (ESI) available. See DOI: $10.1039 / \mathrm{c} 4 \mathrm{sc} 03027 \mathrm{c}$
In contrast, extracellular copper trafficking has been insufficiently investigated due to a lack of appropriate tools for monitoring in living systems. In the more oxidizing extracellular media, $\mathrm{Cu}^{2+}$ is favored over $\mathrm{Cu}^{+} .^{6}$ This oxidized form of copper has long been linked to many neurodegenerative diseases such as Alzheimer's disease, prion diseases, and Parkinson's disease through its participation in the aggregation of fibrogenic proteins. ${ }^{6}$ The protein- $\mathrm{Cu}^{2+}$ interactions are also directly involved in a catalytic cycle of ROS production, which exerts oxidative damage to cells. ${ }^{7}$ Moreover, exogenous copper plays a key role in neuronal signaling pathways by modulating the synaptic plasticity. ${ }^{8}$ Therefore, monitoring dynamic changes in the copper concentration in the extracellular environment will be critical for understanding the mechanisms of $\mathrm{Cu}^{2+}$ release and its physiological functions in the brain. In particular, imaging $\mathrm{Cu}^{2+}$ fluctuations on specifically targeted cell surfaces will greatly improve our insight into cellular responses to outside copper and copper-based cell-to-cell communication. Although a few cellular targeting strategies have been devised for synthetic metal reporters using organelle-targeting moieties, ${ }^{9}$ they cannot direct the probes to specific cells of interest. Furthermore, mislocalization of small-molecular probes inside cells might lead to controversy in data interpretation. ${ }^{\mathbf{1 0}}$ In comparison, genetically encodable protein reporters have major advantages for the cell-specific and surface-specific detection of target molecules. 
Several genetically encoded reporters have been applied for imaging copper in cellular context. ${ }^{\mathbf{1 1}}$ However, they are limited by relatively small responses $(15-50 \%),{ }^{11 b-e}$ low copper selectivity, ${ }^{\mathbf{1 1 a}, \mathbf{1 1 e}}$ or reversed responses in mammalian cells compared with the response in vitro and in living bacteria. ${ }^{11 b}$ Another challenge posed by imaging the extracellular pool of copper in particular is to provide the necessary binding affinity for $\mathrm{Cu}^{2+}$ sensors to compete with copper-binding molecules in the extracellular medium. While extracellular $\mathrm{Cu}^{2+}$ is present at micromolar concentrations (10-25 $\mu \mathrm{M}$ in blood plasma, 0.5-2.5 $\mu \mathrm{M}$ in cerebrospinal fluid (CSF), and $30 \mu \mathrm{M}$ in the synaptic cleft), ${ }^{6}$ the metal ion is rather bound to highly abundant copperbinding proteins or small molecular ligands than present as a free ion. ${ }^{12}$ For example, human serum albumin (HSA), found at $\sim 600 \mu \mathrm{M}$ in plasma, binds $\mathrm{Cu}^{2+}$ with a picomolar binding affinity. ${ }^{13}$ The HSA concentration in CSF is relatively low $(\sim 3$ $\mu \mathrm{M}$ ) but represents $35-80 \%$ of the total CSF proteins. ${ }^{\mathbf{1 4}}$

Developing protein-based $\mathrm{Cu}^{2+}$ sensors with strong but selective copper-binding properties will require potent $\mathrm{Cu}^{2+}$ chelating strategies within proteins. Previously reported sensors have employed simple histidines or a metal-chelating unnatural amino acid (3,4-dihydroxy-L-phenylalanine) to bind $\mathrm{Cu}^{2+}$ since the metal ion prefers to bind nitrogen or oxygen donors. ${ }^{\mathbf{1 1 a}, \mathbf{1 5}}$ Unfortunately, reported binding affinities $\left(K_{\mathrm{D}} \quad 0.2-100 \mu \mathrm{M}\right)$ of these sensors under physiologically relevant conditions have been still insufficient for imaging extracellular $\mathrm{Cu}^{2+}$, and it has not been demonstrated whether they could detect changes in copper concentration under conditions where competing copper-binding biomolecules are present.

Here, we report a new genetically encoded fluorescent reporter that binds $\mathrm{Cu}^{2+}$ with a $K_{\mathrm{D}}$ of $\sim 8 \mathrm{nM}$ while maintaining its selectivity for the metal ion. Green fluorescent protein (GFP) was engineered to contain a natural short but strong $\mathrm{Cu}^{2+}$ binding tripeptide, the amino terminal copper- and nickelbinding (ATCUN) motif, which is also the major copper-binding site of albumins in many species including humans. ${ }^{16}$ The location of this copper binding sequence in GFP was carefully optimized to provide maximum fluorescence quenching, strong $\mathrm{Cu}^{2+}$ binding, and stable protein folding in cells. The resulting reporter was sensitive enough to detect $\mathrm{Cu}^{2+}$ even in the presence of equimolar HSA in vitro. Furthermore, our reporter was stably displayed on cell surfaces and showed specific and reversible responses to extracellular $\mathrm{Cu}^{2+}$.

\section{Results and discussion}

The ATCUN motif consists of three amino acids (Xaa-Xaa-His) with a free N-terminus, where Xaa is any amino acid that can provide an amide nitrogen. As depicted in the X-ray crystal structure, this motif binds $\mathrm{Cu}^{2+}$ by forming a square-planar complex with the $\alpha-\mathrm{NH}_{2}$-terminal nitrogen, the two amide nitrogens, and the imidazole nitrogen in histidine. ${ }^{17}$ Although the peptide binds $\mathrm{Cu}^{2+}\left(K_{\mathrm{D}} \sim 1.18 \times 10^{-16} \mathrm{M}\right)^{18}$ and $\mathrm{Ni}^{2+}\left(K_{\mathrm{D}} \sim\right.$ $\left.10^{-16} \mathrm{M}\right)^{19}$ with similarly high affinities, it is highly context dependent, such that the ATCUN in HSA was reported to bind $\mathrm{Ni}^{2+}$ with a nearly five orders of magnitude weaker binding affinity than $\mathrm{Cu}^{2+} \cdot{ }^{20}$ Considering its small size and strong binding affinity for $\mathrm{Cu}^{2+}$, we hypothesized that the ATCUN motif can serve as a powerful $\mathrm{Cu}^{2+}$-binding site for protein sensors.

To place the ATCUN motif in close proximity of the GFP chromophore, thereby inducing maximum fluorescence quenching by $\mathrm{Cu}^{2+}$ binding, the protein was circularly permuted to generate new $\mathrm{N}$ - and $\mathrm{C}$-termini at the beginning of $\beta$-strand 7 . Circular permutations on $\beta$-strand 7 have shown relatively minimal effects on the GFP fluorescence. ${ }^{21}$ The ATCUN motif (Gly-Gly-His) was then inserted into the $\mathrm{N}$-terminal region in a manner in which the histidine at the third position replaced histidine 148 near the phenolic ring of the chromophore (Fig. 1 and Table 1). To provide an $\alpha-\mathrm{NH}_{2}$-terminal nitrogen for ATCUN in the circularly permuted GFP, a free N-terminus at the first glycine of the motif was precisely generated by intein-based protein cleavage. ${ }^{22} \mathrm{~N}$-terminal sequencing of the intein-cleaved protein confirmed proper insertion of the ATCUN motif (Fig. S1 $\uparrow$ ). For fabrication of the sensor protein, we employed superfolder GFP (OPT) ${ }^{23}$ for higher stability in the assay buffer (100 mM HEPES, pH 7.0, $300 \mathrm{mM} \mathrm{NaCl}$ ) and enhanced folding in $E$. coli as well as in mammalian cells (data not shown).

Fluorescence spectra of the circularly permuted OPT (cpOPT) with the ATCUN insert were acquired by exciting at $480 \mathrm{~nm}$ and collecting the emission signals at 500-600 $\mathrm{nm}$. Upon the addition of $\mathrm{Cu}^{2+}$, the fluorescence intensity of the sensor protein clearly decreased in a concentration-dependent manner (Fig. 2a). The decrease in the fluorescence signal (at $510 \mathrm{~nm}$ ) was up to $<5 \%$ of the apo-protein fluorescence (95\% quenched). Compared with wild-type OPT (wtOPT) and cPOPT, the ATCUNfused cPOPT (GCS-1) exhibited a considerably higher degree of quenching over a wide range of $\mathrm{Cu}^{2+}$ concentrations (Fig. 2b). Although cpOPT displayed noticeable fluorescence quenching at high $\mathrm{Cu}^{2+}$ concentrations $(>10 \mu \mathrm{M})$, introduction of the ATCUN motif increased the $\mathrm{Cu}^{2+}$ sensitivity of the protein. In addition, longer incubation of the ATCUN-fused cpOPT at low $\mathrm{Cu}^{2+}$ concentrations further enhanced fluorescence quenching, which was likely due to the rather slow $\mathrm{Cu}^{2+}$ binding by ATCUN in cpOPT (Fig. 2c). We named the sensor protein as geneticallyencoded copper(II) sensor (GCS)-1 (see Table 1 for a summary of $\mathrm{N}$-terminal sequences in the GCS proteins).

To further optimize the $\mathrm{Cu}^{2+}$ responses, the location of the ATCUN motif in cpOPT was varied around H148 in GCS-1. ATCUN insertion at the -1 position of H148 ((-1)GCS-1, Table

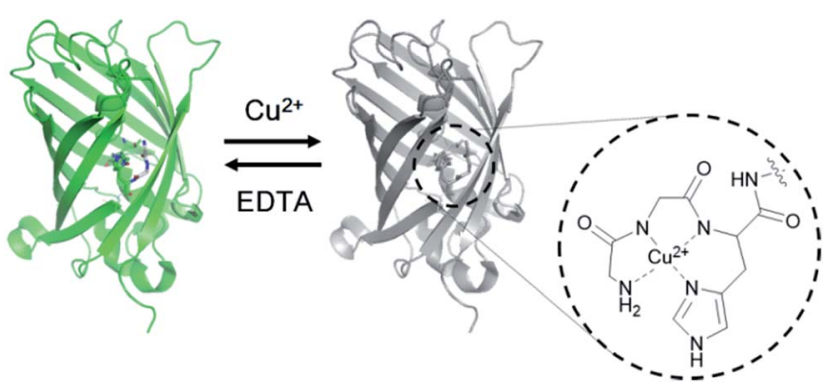

Fig. 1 Design of a genetically encoded fluorescent $\mathrm{Cu}^{2+}$ reporter using the ATCUN motif. Structure of the $\mathrm{Cu}^{2+}$ complex of the ATCUN motif is shown. 
Table $1 \mathrm{~N}$-terminal sequences of GCS and control proteins. The ATCUN motif is shown in bold italic

\begin{tabular}{llllllllllll}
\hline Construct & & & & +1 & $\mathrm{H} 148$ & -1 & & & \\
\hline cpOPT & $\mathrm{NH}_{2}-$ & $\mathrm{E}$ & $\mathrm{L}$ & $\mathrm{S}$ & $\mathrm{H}$ & $\mathrm{N}$ & $\mathrm{V}$ & $\mathrm{Y}$ & $\mathrm{I}$ & $\mathrm{T}$ \\
GCS-1 & & $\mathrm{NH}_{2}-$ & $\boldsymbol{G}$ & $\boldsymbol{G}$ & $\boldsymbol{H}$ & $\mathrm{N}$ & $\mathrm{V}$ & $\mathrm{Y}$ & $\mathrm{I}$ & $\mathrm{T}$ \\
$(-1)$ GCS-1 & & & $\mathrm{NH}_{2}-$ & $\boldsymbol{G}$ & $\boldsymbol{G}$ & $\boldsymbol{H}$ & $\mathrm{V}$ & $\mathrm{Y}$ & $\mathrm{I}$ & $\mathrm{T}$ \\
GCS-2 & & $\mathrm{NH}_{2}-$ & $\boldsymbol{G}$ & $\boldsymbol{G}$ & $\boldsymbol{H}$ & $\mathrm{H}$ & $\mathrm{N}$ & $\mathrm{V}$ & $\mathrm{Y}$ & $\mathrm{I}$ & $\mathrm{T}$ \\
G-GCS-2 & $\mathrm{NH}_{2}-$ & $\mathrm{G}$ & $\boldsymbol{G}$ & $\boldsymbol{G}$ & $\boldsymbol{H}$ & $\mathrm{H}$ & $\mathrm{N}$ & $\mathrm{V}$ & $\mathrm{Y}$ & $\mathrm{I}$ & $\mathrm{T}$ \\
GCS-2-G & & $\mathrm{NH}_{2}-$ & $\boldsymbol{G}$ & $\boldsymbol{G}$ & $\boldsymbol{H}$ & $\mathrm{G}$ & $\mathrm{N}$ & $\mathrm{V}$ & $\mathrm{Y}$ & $\mathrm{I}$ & $\mathrm{T}$
\end{tabular}

(a)
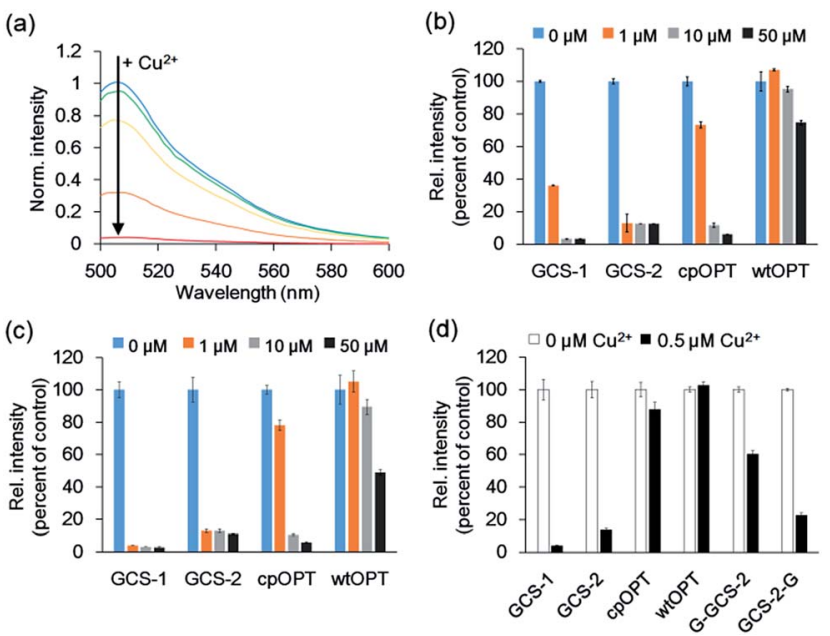

Fig. 2 Fluorescence responses of GCS sensor proteins. (a) Fluorescence responses of $1 \mu \mathrm{M} \mathrm{GCS}-1<5 \mathrm{~min}$ after the addition of $0,0.4,0.8$, 1.6 , and $3.2 \mu \mathrm{M} \mathrm{Cu}^{2+}$. (b) Fluorescence responses of $0.5 \mu \mathrm{M}$ protein $<5$ min after the addition of $0,1,10$, and $50 \mu \mathrm{M} \mathrm{Cu}^{2+}$. cpOPT: circularly permuted green fluorescent protein lacking the ATCUN motif; wtOPT: wild-type green fluorescent protein. (c) Fluorescence responses of $0.5 \mu \mathrm{M}$ protein $1 \mathrm{~h}$ after the addition of $0,1,10$, and $50 \mu \mathrm{M} \mathrm{Cu}^{2+}$. (d) Fluorescence responses of $0.1 \mu \mathrm{M}$ protein $1 \mathrm{~h}$ after the addition of 0 and $0.5 \mu \mathrm{M} \mathrm{Cu}^{2+}$. The error bars correspond to the standard error of the mean of three independent measurements.

1) resulted in a sensor protein with $\mathrm{Cu}^{2+}$ binding responses similar to those of GCS-1 but a poor protein yield (data not shown). In contrast, cpOPT with the ATCUN motif at the +1 position of H148 (GCS-2) showed rapidly saturated fluorescence quenching even at low concentrations of $\mathrm{Cu}^{2+}$ (Fig. 2b and c), possibly indicating a higher $\mathrm{Cu}^{2+}$ binding affinity. Although the degree of quenching was slightly lower for GCS-2 (<15\% of apoprotein fluorescence, $>85 \%$ quenched), the binding response of GCS-2 was significantly faster (within $10 \mathrm{~s}$ ) than that of GCS-1 (>20 min, Fig. S2 $\dagger$ ). The improved response rate of GCS- 2 might be due to an increased flexibility of the ATCUN motif in the protein. Unlike GCS-1, the ATCUN motif in GCS-2 does not include $\mathrm{H} 148$ which is known to interact with neighboring $\beta$ strands. ${ }^{23 a}$ Thereby the ATCUN tripeptide of GCS-2 can be more open for $\mathrm{Cu}^{2+}$ binding, exhibiting a faster association. On the other hand, H148 is also in a hydrogen-bonding network that stabilizes the chromophore. ${ }^{24}$ Conformational changes of $\mathrm{H} 148$ and subsequent effects on the chromophore by $\mathrm{Cu}^{2+}$ binding might be less significant in GCS-2 than in GCS-1. The absorbance spectrum of GCS-1 was altered more strongly by $\mathrm{Cu}^{2+}$ binding than that of GCS-2 (Fig. S3†), possibly indicating stronger influences on the chromophore and thereby a higher degree of quenching response in GCS-1.

To demonstrate that the ATCUN motif is indeed the major $\mathrm{Cu}^{2+}$ binding site in GCS-2, we constructed two GCS-2 variants, G-GCS-2 and GCS-2-G (Table 1). The ATCUN motif in G-GCS-2 was disrupted by adding a glycine residue to the N-terminus of GCS-2. Upon the addition of $\mathrm{Cu}^{2+}$, G-GCS-2 showed a severely diminished quenching response (Fig. 2d). On the other hand, the fluorescence intensity of GCS-2-G, in which the ATCUN motif is intact but $\mathrm{H} 148$ was mutated to glycine, was still reduced to $<30 \%$ of the apo-protein fluorescence $(>70 \%$ quenched) under the same conditions, supporting that the ATCUN motif is primarily responsible for $\mathrm{Cu}^{2+}$ binding (Fig. 2d). In addition, titration with $\mathrm{Cu}^{2+}$ revealed a $1: 1$ binding stoichiometry between GCS- 2 and $\mathrm{Cu}^{2+}$ (Fig. S4 $\dagger$ ), suggesting the presence of a single dominant metal binding site (such as the ATCUN tripeptide) in GCS-2. However, it should be noted that H148 of GCS-2 also plays a partial role in $\mathrm{Cu}^{2+}$ binding and the resulting fluorescence quenching since the $\mathrm{Cu}^{2+}$-mediated response of GCS-2-G was smaller than that of GCS-2.

The metal selectivity of both GCS proteins was subsequently investigated by monitoring changes in fluorescence intensity in the GCS proteins when other biologically relevant metal ions $\left(\mathrm{Cr}^{3+}, \mathrm{Mn}^{2+}, \mathrm{Fe}^{3+}, \mathrm{Co}^{2+}, \mathrm{Ni}^{2+}, \mathrm{Zn}^{2+}, \mathrm{Ag}^{+}, \mathrm{Cd}^{2+}, \mathrm{Hg}^{2+}, \mathrm{Pb}^{2+}, \mathrm{K}^{+}\right.$, $\mathrm{Ca}^{2+}$, and $\mathrm{Mg}^{2+}$ ) were present. Most of these metal ions had no effect on the fluorescence quenching by $\mathrm{Cu}^{2+}$ (Fig. 3a and b). Although $\mathrm{Ni}^{2+}$ caused a small fluorescence change for GCS-2, subsequent $\mathrm{Cu}^{2+}$ addition readily induced fluorescence quenching (Fig. 3b). The ATCUN motif in our GCS sensor proteins likely binds to $\mathrm{Ni}^{2+}$ more weakly than to $\mathrm{Cu}^{2+}$, which is similar to the ATCUN motif in HSA. ${ }^{20}$ The effects of $\mathrm{Cu}^{+}$were examined in a buffer containing a 50-fold molar excess of L-ascorbic acid. L-ascorbic acid had no influence on the fluorescence intensities of the GCS proteins (Fig. S5†) or $\mathrm{Cu}^{2+}$ binding of the ATCUN motifs. ${ }^{25} \mathrm{Cu}^{+}$addition showed no change in fluorescence for both GCS proteins (Fig. 3c). The reversibility of the fluorescence quenching of the sensor protein was also tested, and fluorescence signals from the $\mathrm{Cu}^{2+}$-bound GCS proteins were recovered upon addition of metal-chelating ethylenediaminetetraacetic acid (EDTA, Fig. 3d).

The $\mathrm{Cu}^{2+}$-binding affinities of the GCS proteins were determined by measuring fluorescence changes upon titration with copper. ${ }^{15 b}$ All assays were performed at relatively high buffer and salt concentrations (100 mM HEPES, pH 7.0, $300 \mathrm{mM}$ $\mathrm{NaCl}$ ), which might reflect various extracellular media. ${ }^{26}$ The dissociation constant for GCS-2 was calculated to be $7.93 \pm 3.20$ nM (Fig. 4a), which is the strongest $\mathrm{Cu}^{2+}$ binding among the currently available protein-based $\mathrm{Cu}^{2+}$ sensors (Table $\mathrm{S} 1 \dagger$ ). The use of a natural copper(II)-specific tripeptide provides a strong chelating strategy for robust $\mathrm{Cu}^{2+}$ capture near the GFP chromophore, presenting high sensitivity for the metal ion while not compromising the metal selectivity of the GCS proteins. GCS-1 was found to bind $\mathrm{Cu}^{2+}$ more weakly $(94.04 \pm 19.33 \mathrm{nM})$ as discussed above (Fig. S6†). 
(a)

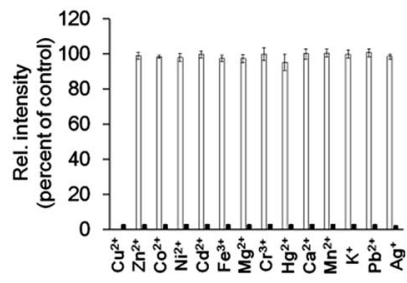

(c)

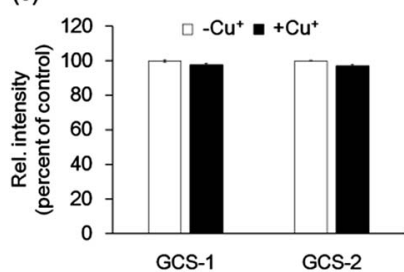

(b)

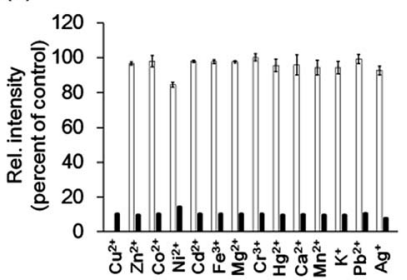

(d)

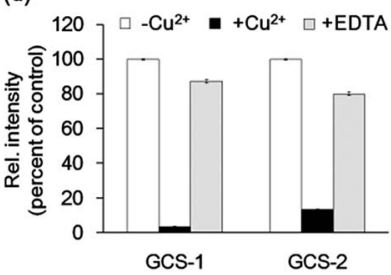

Fig. $3 \mathrm{Cu}^{2+}$ selectivity and reversibility of GCS proteins. ( $a$ and $b$ ) Fluorescence responses of $1 \mu \mathrm{M} \mathrm{GCS}-1$ (a) and GCS-2 (b) to various metal ions. White bars represent fluorescence responses with $5 \mu \mathrm{M}$ of the respective metal ions. Black bars represent fluorescence responses after the addition of $5 \mu \mathrm{M} \mathrm{Cu}^{2+}$. (c) Fluorescence responses of $1 \mu \mathrm{M}$ GCS protein with $5 \mu \mathrm{M} \mathrm{Cu}^{+}$including $250 \mu \mathrm{M}$ of L-ascorbic acid to prevent oxidation of $\mathrm{Cu}^{+}$to $\mathrm{Cu}^{2+}$. Fluorescence spectra were collected within 5 min after $\mathrm{Cu}^{+}$addition. (d) Fluorescence responses of $1 \mu \mathrm{M}$ GCS proteins with $5 \mu \mathrm{MCu}^{2+}$ followed by the addition of $50 \mu \mathrm{M}$ EDTA. All responses were normalized against the fluorescence intensity of $1 \mu \mathrm{M}$ GCS protein lacking a metal ion. The error bars correspond to the standard error of the mean of three independent measurements.

To test whether the binding affinity of the GCS proteins for $\mathrm{Cu}^{2+}$ is high enough to monitor the metal ion even in the presence of other copper-binding molecules, the GCS responses to $\mathrm{Cu}^{2+}$ were measured in the presence of various amounts of HSA. GCS-2 showed $\mathrm{Cu}^{2+}$ binding properties comparable to HSA, where $>40 \%$ of the added $\mathrm{Cu}^{2+}$ was bound to GCS- 2 in the presence of equimolar HSA (Fig. 4b). Considering that the reported dissociation constants for HSA are in the picomolar range, ${ }^{13} \mathrm{Cu}^{2+}$ binding to GCS-2 should be weaker by at least three orders of magnitude. This discrepancy is likely due to the different experimental methods used for $K_{\mathrm{D}}$ measurements, components/concentration of buffers, $\mathrm{pH}$, or ionic strengths. ${ }^{27}$ Nevertheless, under our conditions, an optimized ATCUN tripeptide in GCS-2 showed nearly as strong binding as the ATCUN motif in HSA. In contrast, the fluorescence intensity of GCS-1 began to decrease only after the concentration of $\mathrm{Cu}^{2+}$ was high enough to fill in most of the ATCUN sites in HSA (Fig. 4c). Therefore, GCS-1 was not able to effectively compete with HSA. We also performed competing experiments with an amyloid beta peptide (A $\beta 40)$, another copper-binding molecule $\left(K_{\mathrm{D}} 0.47-\right.$ $30 \mu \mathrm{M})$ which plays a key role in the pathogenesis of Alzheimer's disease. ${ }^{28} \mathrm{~A} \beta 40$, which has a rather weak $\mathrm{Cu}^{2+}$-binding ability, was outcompeted by both GCS proteins (Fig. S7†). Taken together, our data show that GCS-2 $\left(K_{\mathrm{D}} \sim 8 \mathrm{nM}\right)$ is a highly sensitive reporter that can efficiently compete with other highaffinity copper-binding molecules in extracellular media. In comparison, GCS-1 ( $\left.K_{\mathrm{D}} \sim 94 \mathrm{nM}\right)$ might be useful for applications in which low-affinity reporter proteins are required.

(a)

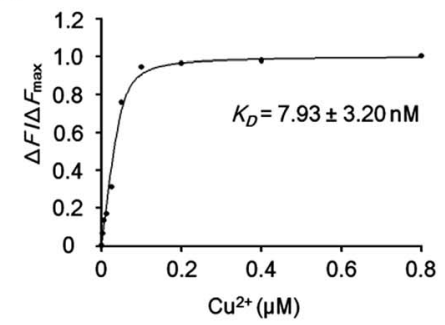

(b)

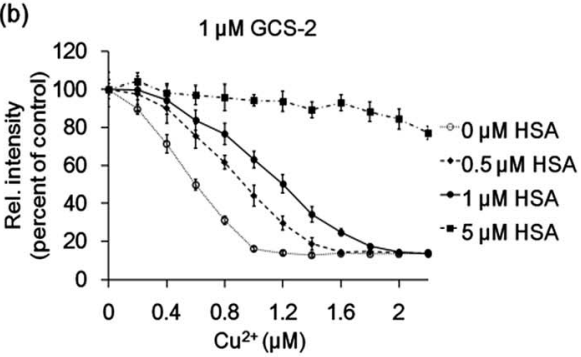

(c)

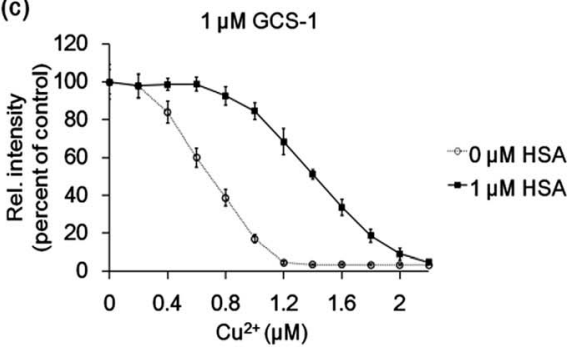

Fig. 4 (a) Representative plot of $\Delta F / \Delta F_{\max }$ of $50 \mathrm{nM} \mathrm{GCS}-2$ with various $\mathrm{Cu}^{2+}$ concentrations. The binding constant $K_{\mathrm{D}}$ was derived from three independent experiments. $\Delta F$ : change in measured fluorescence, $\Delta F_{\text {max }}$ : maximum fluorescence change. (b) GCS-2 fluorescence changes with varying concentration of $\mathrm{Cu}^{2+}$ in the absence and presence of $0.5,1$, and $5 \mu \mathrm{M} \mathrm{HSA}$. (c) GCS-1 fluorescence changes with varying concentration of $\mathrm{Cu}^{2+}$ in the absence and presence of $1 \mu \mathrm{M}$ HSA. The error bars correspond to the standard error of the mean of three independent measurements.

Finally, we used the GCS proteins to detect dynamic $\mathrm{Cu}^{2+}$ fluctuations on the surfaces of live mammalian cells. A signal peptide for the secretory pathway and the transmembrane domain of the platelet-derived growth factor receptor were fused to GCS-2 for display on the extracellular plasma membrane. The signal peptide is cleaved during sensor protein synthesis in the endoplasmic reticulum, generating the ATCUN motif precisely at the $\mathrm{N}$-terminus for effective $\mathrm{Cu}^{2+}$ binding. HeLa cells expressing the GCS-2 construct were monitored with a total internal reflection fluorescence (TIRF) microscope for selective visualization of the cell surface fluorescence. Upon $\mathrm{Cu}^{2+}$ addition $(50 \mu \mathrm{M})$, the GCS- 2 fluorescence intensity on the cell surface was rapidly decreased within $\sim 1.5 \mathrm{~min}$, and it was saturated to $47 \%$ (average from 13 cells in the same well, 53\% quenched) of the initial signal after $3 \mathrm{~min}$ (Fig. 5). EDTA was then added to the cells, and the GCS-2 fluorescence intensity was recovered to $97 \%$ within $4 \mathrm{~min}$, which is in agreement with the in vitro results. GCS-1 that was expressed on HeLa cell surfaces provided fluorescence changes similar to those of GCS2 against extracellular $\mathrm{Cu}^{2+}$ (data not shown). 
(a)
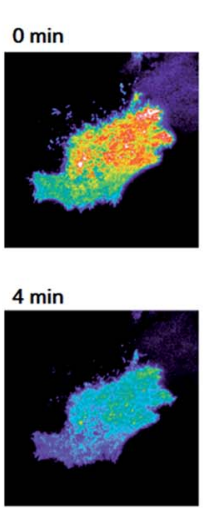

$+50 \mu \mathrm{M} \mathrm{CuCl}_{2}$ 1 min

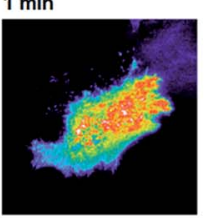

+1 mM EDTA 1 min

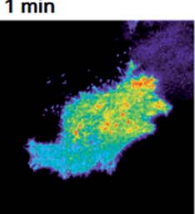

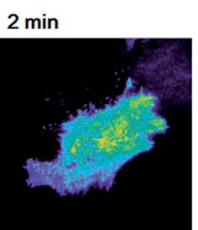

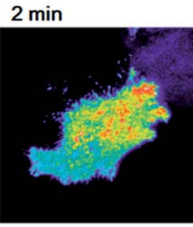

$3 \mathrm{~min}$
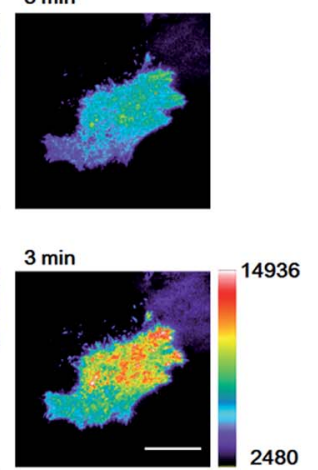

(b)

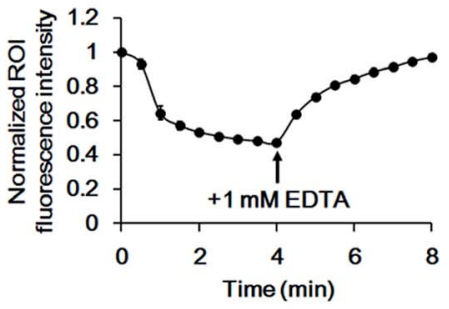

Fig. 5 Imaging of $\mathrm{Cu}^{2+}$ on live HeLa cell surfaces by GCS-2. (a) Pseudocolored images from 1 min prior to $\mathrm{Cu}^{2+}$ addition to $1,2,3$ and 4 min after the addition of $50 \mu \mathrm{M} \mathrm{Cu}^{2+}$, and 1, 2 and 3 min after the addition of $1 \mathrm{mM}$ EDTA. Images were collected using total internal reflection fluorescence (TIRF) microscopy. The color bar represents the fluorescence intensity in the GFP channel (excitation at $488 \mathrm{~nm}$ ). Scale bar: $20 \mu \mathrm{m}$. (b) Plot of the average fluorescence response of 13 cells vs. time after $50 \mu \mathrm{M} \mathrm{Cu}^{2+}$ addition. All fluorescence intensity values were subtracted by background signals and normalized by the fluorescence before $\mathrm{Cu}^{2+}$ addition. The time of $1 \mathrm{mM}$ EDTA addition is shown. The error bars correspond to the standard error of the mean value of 13 individual cells.

To confirm that the decrease in fluorescence of GCS-2 was specific for $\mathrm{Cu}^{2+}$ binding, $\mathrm{Zn}^{2+}$, another crucial metal ion in the brain, was added to the cells $(50 \mu \mathrm{M})$ before $\mathrm{Cu}^{2+}$ treatment.

(a)

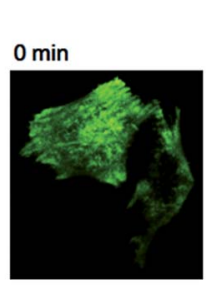

$+50 \mu \mathrm{MZnCl}_{2}$



$+50 \mu \mathrm{MCuCl}_{2}$
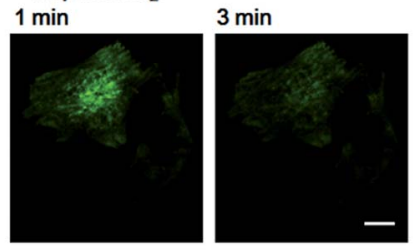

(b)

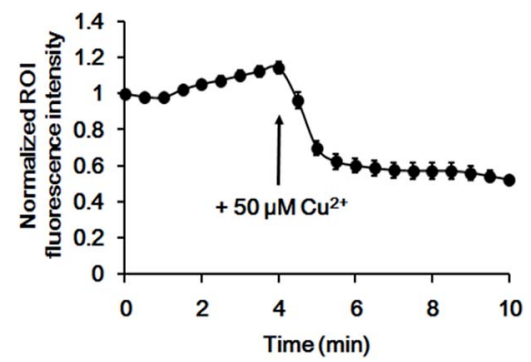

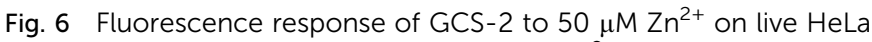
cell surfaces followed by addition of $50 \mu \mathrm{M} \mathrm{Cu}^{2+}$. (a) Images of $1 \mathrm{~min}$ prior to $\mathrm{Zn}^{2+}$ addition, $3 \mathrm{~min}$ after the addition of $\mathrm{Zn}^{2+}$, and 1 and $3 \mathrm{~min}$ after the addition of $\mathrm{Cu}^{2+}$. Scale bar: $10 \mu \mathrm{m}$. (b) Plot of the average

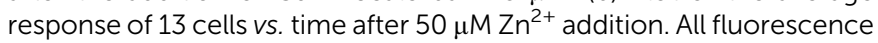
intensity values were subtracted by background signals and normalized by the fluorescence before $\mathrm{Zn}^{2+}$ addition. The time of $50 \mu \mathrm{M} \mathrm{Cu}^{2+}$ addition is shown. The error bars correspond to the standard error of the mean value of 13 individual cells.
Interestingly, the fluorescence intensity of several cells of the 13 monitored cells was slightly increased $(<115 \%)$ by $\mathrm{Zn}^{2+}$ (Fig. 6). This could result from structural stabilization of the $\beta$-barrel of (a)

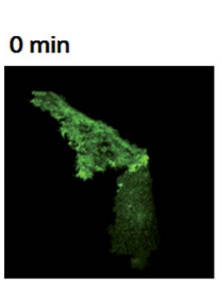

$+10 \mu \mathrm{MCuCl}_{2}$ $4 \mathrm{~min}$

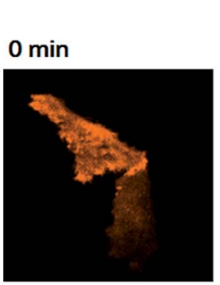

$4 \mathrm{~min}$

(b)

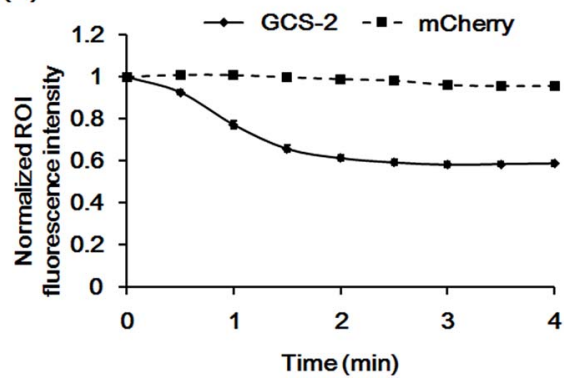

Fig. 7 Fluorescence responses of GCS-2 and mCherry co-expressed in HeLa cells to $10 \mu \mathrm{M} \mathrm{Cu}^{2+}$. (a) Images of GCS-2 (top) and mCherry (bottom) of $1 \mathrm{~min}$ prior to $\mathrm{Cu}^{2+}$ addition and $4 \mathrm{~min}$ after the addition of $\mathrm{Cu}^{2+}$. Scale bar: $10 \mu \mathrm{m}$ (b) plots of the average fluorescence responses of GCS -2 and mCherry of 8 cells vs. time after $10 \mu \mathrm{M} \mathrm{Cu}^{2+}$ addition. All fluorescence intensity values were subtracted by background signals and normalized by the fluorescence before $\mathrm{Cu}^{2+}$ addition. The error bars correspond to the standard error of the mean value of eight individual cells. 
the GCS-2 protein under relatively high zinc concentrations as recently reported for a red fluorescent mKate2 mutant. ${ }^{11 a}$ Zin$\mathrm{c}$ (II)-mediated changes in protein distribution in the plasma membrane might also affect the fluorescence intensity of GCS-2 as observed using TIRF imaging. Nevertheless, all cells exhibited rapid fluorescence quenching upon $\mathrm{Cu}^{2+}$ addition (Fig. 6). The copper(II)-specific fluorescence decrease for GCS-2 was further confirmed by its co-expression with mCherry, a red fluorescent protein, on the cell surfaces. Addition of $10 \mu \mathrm{M}$ $\mathrm{CuCl}_{2}$ decreased the fluorescence intensity of GCS-2 but not that of mCherry, which again shows the applicability of GCS-2 for specific $\mathrm{Cu}^{2+}$ imaging on the surface of live cells (Fig. 7). Moreover, the fluorescence signals of cpOPT proteins on cell surfaces were not altered by $10 \mu \mathrm{M} \mathrm{Cu}^{2+}$ (Fig. S8 $\dagger$ ).

\section{Conclusions}

The GCS proteins demonstrate that the natural and small $\mathrm{Cu}^{2+}$ binding ATCUN motif can be successfully utilized as a strong and specific copper chelating site for genetically encoded $\mathrm{Cu}^{2+}$ reporters. The tight $\mathrm{Cu}^{2+}$ binding of GCS-2 allows for reliable $\mathrm{Cu}^{2+}$ monitoring in the presence of HSA, one of the major $\mathrm{Cu}^{2+}$ binding biomolecules in extracellular environments. The sensor proteins also demonstrate high selectivity for $\mathrm{Cu}^{2+}$ compared with other biologically relevant metal ions. Imaging $\mathrm{Cu}^{2+}$ on the surface of live cells with GCS-2 showed a quick and reversible response, which demonstrates the possibility of monitoring oscillating $\mathrm{Cu}^{2+}$ levels in living systems. The copper-chelating strategy described herein has multiple advantages. Taken from one of the major players in extracellular copper trafficking, the ATCUN motif may enable noninvasive detection of dynamic changes in copper concentration in biological systems. The motif is composed of only three amino acids, creating minimal disruption in the fluorescent protein's structure. To expand the applicability of GCS sensors to intracellular $\mathrm{Cu}^{2+}$ imaging, strategies to generate ATCUN motifs and protect these motifs from various post-translational modifications ${ }^{29}$ inside cells should be developed. Nonetheless, we envision that GCS proteins can be employed to monitor $\mathrm{Cu}^{2+}$ release on neuronal cells. Specifically, copper release in the synaptic cleft which is induced by chemical or biological cues could be detected by GCS proteins expressed on pre- or postsynaptic cell surfaces. Further studies will be aimed at improving the membrane targeting efficiency and applications of red fluorescent proteins that enable $\mathrm{Cu}^{2+}$ imaging in live animals.

\section{Acknowledgements}

This work was supported by the Basic Science Research Program through the National Research Foundation of Korea (NRF) funded by the Ministry of Science, ICT \& Future Planning (MSIP) (2011-0020322) and BioNano Health Guard Research Center funded by MSIP as Global Frontier Project (HGUARD_2014M3A6B2060512 \& 2013M3A6B2078950).

\section{Notes and references}

1 B. E. Kim, T. Nevitt and D. J. Thiele, Nat. Chem. Biol., 2008, 4, 176-185.

2 B. Halliwell and J. M. Gutteridge, Biochem. J., 1984, 219, 114.

3 E. Madsen and J. D. Gitlin, Annu. Rev. Neurosci., 2007, 30, 317-337.

4 (a) K. P. Carter, A. M. Young and A. E. Palmer, Chem. Rev., 2014, 114, 4564-4601; (b) D. W. Domaille, E. L. Que and C. J. Chang, Nat. Chem. Biol., 2008, 4, 168-175.

5 E. M. Rees and D. J. Thiele, J. Biol. Chem., 2007, 282, 2162921638.

6 E. L. Que, D. W. Domaille and C. J. Chang, Chem. Rev., 2008, 108, 1517-1549.

7 E. Gaggelli, H. Kozlowski, D. Valensin and G. Valensin, Chem. Rev., 2006, 106, 1995-2044.

8 E. D. Gaier, B. A. Eipper and R. E. Mains, J. Neurosci. Res., 2013, 91, 2-19.

9 (a) R. J. Radford, W. Chyan and S. J. Lippard, Chem. Sci., 2014, 5, 4512-4516; (b) Y. H. Lee, N. Park, Y. B. Park, Y. J. Hwang, C. Kang and J. S. Kim, Chem. Commun., 2014, 50, 3197-3200; (c) R. J. Radford, W. Chyan and S. J. Lippard, Chem. Sci., 2013, 4, 3080-3084; (d) S. Iyoshi, M. Taki and Y. Yamamoto, Org. Lett., 2011, 13, 4558-4561; (e) D. Li, S. Chen, E. A. Bellomo, A. I. Tarasov, C. Kaut, G. A. Rutter and W. H. Li, Proc. Natl. Acad. Sci. U. S. A., 2011, 108, 21063-21068; (f) S. C. Dodani, S. C. Leary, P. A. Cobine, D. R. Winge and C. J. Chang, J. Am. Chem. Soc., 2011, 133, 8606-8616.

10 K. A. Price, J. L. Hickey, Z. G. Xiao, A. G. Wedd, S. A. James, J. R. Liddell, P. J. Crouch, A. R. White and P. S. Donnelly, Chem. Sci., 2012, 3, 2748-2759.

11 (a) X. Yu, M. P. Strub, T. J. Barnard, N. Noinaj, G. Piszczek, S. K. Buchanan and J. W. Taraska, PLoS One, 2014, 9, e95808; (b) J. Liu, J. Karpus, S. V. Wegner, P. R. Chen and C. He, J. Am. Chem. Soc., 2013, 135, 3144-3149; (c) M. S. Koay, B. M. Janssen and M. Merkx, Dalton Trans., 2013, 42, 3230-3232; (d) J. Liang, M. Qin, R. Xu, X. Gao, Y. Shen, Q. Xu, Y. Cao and W. Wang, Chem. Commun., 2012, 48, 3890-3892; (e) S. V. Wegner, H. Arslan, M. Sunbul, J. Yin and C. He, J. Am. Chem. Soc., 2010, 132, 2567-2569.

12 J. H. Kaplan and S. Lutsenko, J. Biol. Chem., 2009, 18, 2546125465.

13 M. Rozga, M. Sokolowska, A. M. Protas and W. Bal, J. Biol. Inorg.Chem., 2007, 12, 913-918.

14 H. Reiber, Clin. Chim. Acta, 2001, 310, 173-186.

15 (a) E. É. Bálint, J. Petres, M. Szabó, C. K. Orbán, L. Szilágyi and B. Ábrahám, J. Fluoresc., 2013, 23, 273-281; (b) N. Ayyadurai, N. S. Prabhu, K. Deepankumar, S.-G. Lee, H.-H. Jeong, C.-S. Lee and H. Yun, Angew. Chem., Int. Ed., 2011, 50, 6534-6537; (c) T. A. Richmond, T. T. Takahashi, R. Shimkhada and J. Bernsdorf, Biochem. Biophys. Res. Commun., 2000, 268, 462-465.

16 C. Harford and B. Sarkar, Acc. Chem. Res., 1997, 30, 123-130. 
17 N. Camerman, A. Camerman and B. Sarkar, Can. J. Chem., 1976, 54, 1309-1316.

18 S. J. Lau, T. P. Kruck and B. Sarkar, J. Biol. Chem., 1974, 249, 5878-5884.

19 E. C. Long, Acc. Chem. Res., 1999, 32, 827-836.

20 W. Bal, M. Sokolowska, E. Kurowska and P. Faller, Biochim. Biophys. Acta, 2013, 1830, 5444-5455.

21 G. S. Baird, D. A. Zacharias and R. Y. Tsien, Proc. Natl. Acad. Sci. U. S. A., 1999, 96, 11241-11246.

22 S. Chong, K. S. Williams, C. Wotkowicz and M. Q. Xu, J. Biol. Chem., 1998, 273, 10567-10577.

23 (a) J. D. Pedelacq, S. Cabantous, T. Tran, T. C. Terwilliger and G. S. Waldo, Nat. Biotechnol., 2006, 24, 79-88; (b)
S. Cabantous, T. C. Terwilliger and G. S. Waldo, Nat. Biotechnol., 2005, 23, 102-107.

24 M. A. Elsliger, R. M. Wachter, G. T. Hanson, K. Kallio and S. J. Remington, Biochemistry, 1999, 38, 5296-5301.

25 Y. Jin and J. A. Cowan, J. Am. Chem. Soc., 2005, 127, 84088415.

26 G. G. Somjen, in Ions in the Brain: Normal Function, Seizures, and Stroke, Oxford University Press, Inc, New York, 2004, p. 16.

27 Y. Zhang, S. Akilesh and D. E. Wilcox, Inorg. Chem., 2000, 39, 3057-3064.

28 V. Tougu, A. Karafin and P. Palumaa, J. Neurochem., 2008, 104, 1249-1259.

29 T. Meinnel and C. Giglione, Proteomics, 2008, 8, 626-649. 